\title{
Rohstoffe und Bildungsaktivitäten des RIC Leoben
}

\author{
Ulrike Haslinger und Anja Meier \\ Resources Innovation Center Leoben, Montanuniversität Leoben, Leoben, Österreich \\ Eingegangen 28. Januar 2019; angenommen 3. Februar 2019; online publiziert 18. Februar 2019
}

\begin{abstract}
Zusammenfassung: Die Montanuniversität Leoben beteiligt sich mit dem Resources InnovationCenter (RIC) Leoben seit einigen Jahren maßgeblich an der Entwicklung von Bildungsaktivitäten des EIT RM und liefert einen umfassenden und qualitätsvollen Beitrag zum Austausch fundierter, fachlicher und angewandter Expertise von der Primar- und Sekundarstufe über die tertiäre bis hin zur berufsbegleitenden Bildung. Zu den Bildungsschwerpunkten gehören die Nachwuchsförderung in den naturwissenschaftlichen Fächern, die Förderung der Bildungsqualität im ost- und südosteuropäischen Raum, Bewusstseinsbildung und Training transversaler Fertigkeiten für Studierende und Lehrende, die Stärkung unternehmerischer Bildungsinhalte in der Ausbildung, die Digitalisierung in der Bildung sowie die Internationalisierung der Montanuniversität Leoben. In diesem Beitrag wird ein Einblick in den vielfältigen und umfangreichen Arbeitsbereich des RIC Leoben gegeben.
\end{abstract}

Schlüsselwörter: Rohstoffsektor, Hochschulbildung, Internationalisierung, Lehre, Digitalisierung,

Bildungsbedarf, EIT, Innovation, Bewusstseinsbildung,

Rohstoffe, Ressourcen, Nachhaltigkeit, Kreislaufwirtschaft, Lebenslanges Lernen, Weiterbildung,

Erwachsenenbildung, Wissenschaft, Industrie, Forschung

\section{Education in Raw Materials}

Abstract: For some years now, Montanuniversitaet Leoben and Resources Innovation Center (RIC) Leoben have been deeply involved in EIT RM's educational activities delivering significant and high-quality contributions to the exchange of profound subject matter expertise and applied know-how from primary and secondary to tertiary levels, including professional education. Particular focus is put on the support and the promotion of young people in the natural sciences, boosting high-quality education in east and

\footnotetext{
Mag. phil. Dr. phil. U. Haslinger ( $\varangle$ )

Resources Innovation Center Leoben,

Montanuniversität Leoben,

Franz-Josef-Straße 18,

8700 Leoben, Österreich

ulrike.haslinger@unileoben.ac.at
}

southeast European countries, awareness raising for and training of transversal skills for both students and academic staff, strengthening of entrepreneurial aspects and digitalisation in education, and fostering the internationalisation of Montanuniversitaet Leoben. This article gives insight into the diverse, broad-based work area of RIC Leoben.

Keywords: Raw Materials, Higher Education, Internationalisation, Teaching, Digitisation, EIT, Innovation, Entrepreneurship, Resources, Sustainability, Circular Economy, Life-long learning, Continuing education, Science, Industry, Research

\section{Das EIT RawMaterials (EIT RM), das Resources and Innovation Center Leoben (RIC Leoben) und ihre Aktivitäten für den internationalen Bildungsbereich}

Für die Aus- und Weiterbildung im Rohstoffsektor hat das EIT RM den Bereich „Education“ eingerichtet, der sich in den vergangenen Jahren durch großes Engagement und umfangreiche Investitionen als eine bedeutende Säule in der europäischen Bildungslandschaft etabliert hat. Ziel der zahlreichen Bildungsaktivitäten des EIT RM ist es, dem Mangel an qualifizierten Arbeitskräften im Rohstoffsektor entgegen zu wirken, der von der Europäischen Union in ihrem Bericht European Innovation Partnership on Raw Materials. Raw Materials Scoreboard [1] erfasst wurde. Um dabei die speziellen Bildungsbedarfe zu identifizieren, führte das EIT RM 2016 eine Umfrage durch (Survey 611443 "Industrial needs of continuous education in the EIT RawMaterials“), deren Ergebnisse die Grundlage zur Entwicklung vieler EIT RM Projekte in Forschung, Industrie und Schule bilden.

Die Montanuniversität Leoben beteiligt sich bereits seit einigen Jahren an den Bildungsaktivitäten des EIT RM. Dabei hat sich speziell das RIC Leoben zu einem wichtigen Partner des EIT RM entwickelt, der einen umfassenden und qualitätsvollen Beitrag zum Austausch fundierter, fachlicher und angewandter Expertise von der Primar- und Sekundarstufe über die tertiäre bis hin zur berufsbegleitenden Bil- 
dung leistet. Im Folgenden wird ein Einblick in den vielfältigen und umfangreichen Arbeitsbereich des RIC Leoben gegeben.

Besondere Aufmerksamkeit wird der ost- und südosteuropäischen (East and Southeast Europe/ESEE) Bildungslandschaft gewidmet, die durch Kooperationen mit Bildungseinrichtungen im schulischen und universitären Bereich sowie mit Industriepartnern gestärkt werden soll. Beispiele der guten Praxis sollen Orientierung und Raum zur Zusammenarbeit geben. Ausgehend vom Wunsch ostund südosteuropäischer Partnerinstitutionen, das Angebot der Bildungseinrichtungen im ESEE-Raum international wettbewerbsfähiger zu gestalten, zählen hier unter Berücksichtigung der europäischen Bildungsziele ( „European Higher Education Area and Bologna process", http://www. ehea.info [2]) zu den zentralen Herausforderungen die Förderung hochwertiger Bildung im ESEE-Raum und ihre stärkere Vernetzung mit regionalen Industriepartnern, sodass hochqualifizierten Personen in diesen Ländern Alternativen zur beruflich motivierten Abwanderung geboten werden können.

Seiner geografischen Lage und seinen Beiträgen zur Erweiterung von Ausbildungs- und Beschäftigungsmöglichkeiten im ESEE-Raum verdankt daher das RIC Leoben 2017 die Ernennung zur „Education Platform East”, welche als strategische Schnittstelle wichtige Partner in der Region vernetzt und zentrale EIT RM Aktivitäten leitet und implementiert. Dazu zählen zum Beispiel die jährliche Durchführung von „Education Dialogue/ESEE Dialogue Conferences" und die Teilnahme als ordentliches Mitglied in der "ESEE Education Strategy Group", welche zur Entwicklung der Bildungsqualität im ESEE-Raum eingerichtet wurde. In dieser Arbeitsgruppe ist jedes Mitglied neben der Mitwirkung an einem umfangreichen strategischen Bildungskonzept auch für die unmittelbare Umsetzung konkreter Maßnahmen verantwortlich.

Das RIC Leoben widmet sich jährlich neuen Bildungsschwerpunkten. Zu den aktuellen Themen gehören die Nachwuchsförderung in den naturwissenschaftlichen Fächern, (wie eingangs erwähnt) die Förderung der Bildungsqualität im ESEE-Raum, Bewusstseinsbildung und Training transversaler Fertigkeiten für Studierende und Lehrende, die Stärkung unternehmerischer Bildungsinhalte in der Ausbildung, die Digitalisierung in der Bildung sowie die Internationalisierung der Montanuniversität Leoben.

Darüber hinaus ist das RIC Leoben die Anlaufstelle für Informationen rund um das EIT RM an der Montanuniversität Leoben. Beispielsweise informiert und begleitet das RIC Leoben die Lehrstühle in der Antragsphase zur jährlichen Ausschreibung des EIT RM „Kick Added Value Activities“ (KAVA) in den Kategorien „Education”, "RIS/INT“ und "Upscaling“ und kooperiert mit den Lehrstühlen bei der Durchführung von Projekten. Die KAVA-Projekte des EIT RM, die sich im Portfolio des RIC Leoben befinden, sind der Bildungssäule "Learning \& Outreach" zuzuordnen. Sie umfassen die Kategorien "Wider Society Learning" "Lifelong Learning", "Master Programme" und "PhD Programme" und decken dabei Bildungsbedarfe von der Primar- und Sekundarstufe bis hin zur Hochschulbildung sowie des Lebenslangen Lernens ab.
Obwohl internationale Mobilitätsprogramme traditioneller Weise vom MIRO, dem Montanuniversitaet International Relations Office, betreut werden, organisiert das RIC Leoben den internationalen Austausch von Studierenden und Lehrenden im Rahmen des CEEPUS-Netzwerks. Dabei handelt es sich um ein thematisches Netzwerk mit dem Titel "Raw Materials Smart Innovation Strategies in ESEE Region“ (CEEPUS RAMSIS), das speziell für den akademischen Austausch im Rohstoffsektor eingerichtet wurde. CEEPUS RAMSIS umfasst derzeit acht Partnerländer: Bulgarien, Kroatien, Mazedonien, Österreich, Polen, die Slowakei, Serbien und Ungarn und wird von Österreich (vom RIC Leoben) geleitet. Das Programm bietet Mobilitäten für kurz- und langfristige Aufenthalte sowie finanzielle Unterstützung bei kurzfristigen Exkursionen und Sommeruniversitäten in den teilnehmenden Partnerländern.

Durch die Finanzierung von Bildungsprojekten anerkennt das EIT RM, dass so früh wie möglich und durch kontinuierliche Maßnahmen und Aktivitäten der Weg für einen nachhaltigen und bewussten Umgang mit Rohstoffen in unserer Gesellschaft bereitet werden muss. In diesem Zusammenhang sind naturwissenschaftliche, technische, ökologische, unternehmerische, wirtschaftliche und soziale Aspekte des Bergbaus zu verstehen und daher nimmt die Bedeutung eines interdisziplinären Zugangs zu Bildungsinhalten, welcher die Lernenden auf eine hochtechnologisierte und nachhaltige Industrie vorbereiten soll, kontinuierlich zu.

Dabei gewinnen transversale Fertigkeiten immer mehr an Gewicht. Obwohl selbstverständlich der Wissenstransfer das zentrale Element einer jeden Ausbildung ist, steht außer Zweifel, dass ein Individuum über ein weitaus breiteres Repertoire an zusätzlichen Kompetenzen und Fertigkeiten verfügen muss, um in seinem professionellen Umfeld erfolgreich zu sein und um sich den kontinuierlich entwickelnden Herausforderungen auf dem Arbeitsmarkt stellen zu können. Dazu zählen im Sinne einer interdisziplinären Ausbildung im Rohstoffsektor unter anderem unternehmerische Fertigkeiten, kommunikative Fertigkeiten (wie zum Beispiel fremdsprachliche, fachsprachliche, rhetorische Kompetenzen), Präsentationsfertigkeiten, akademisches Schreiben für Publikationen, Projektmanagement, Evaluierungskompetenzen, Marketingkompetenzen und pädagogische Kompetenzen wie angewandte Didaktik und Methodik in Wissenschaft und Technik. Neue Kooperationspartner, wie zum Beispiel das Europäische Fremdsprachenzentrum des Europarats (www.ecml.at), mit dem das RIC Leoben eine Weiterbildung im Bereich transversaler Fertigkeiten plant, stützen den interdisziplinären Bildungsansatz der Montanuniversität Leoben.

Letztlich werden diese Fertigkeiten und Kompetenzen auch für die zunehmende Internationalisierung in der Bildung benötigt, mit welcher Universitäten Schritt halten müssen, um durch hohe Bildungsstandards international wettbewerbsfähig zu bleiben. Dabei müssen mitunter sprachliche Anforderungen sowie interkulturelle Kompetenzen erfüllt werden, um sich in internationalen Settings sowohl in der Bildung als auch im professionellen Umfeld erfolgreich behaupten zu können. Die Aktivitäten des RIC Leoben im Bereich der Internationalisierung haben daher 


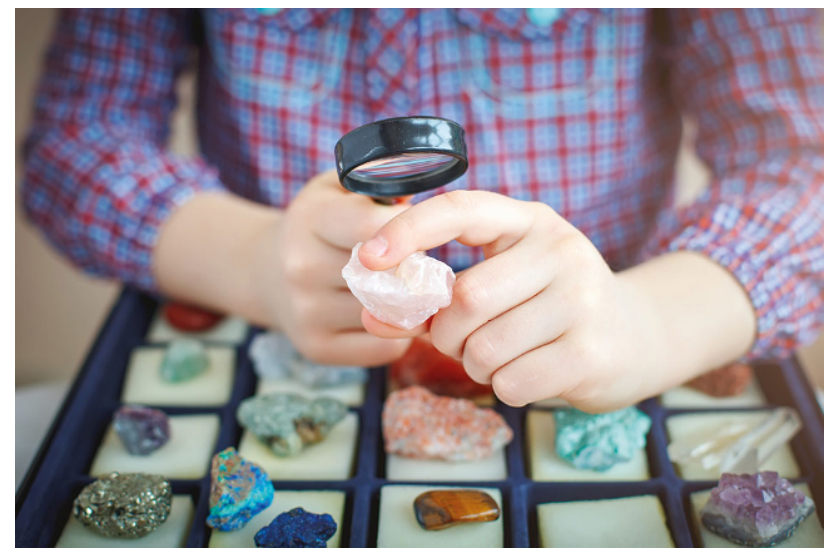

Abb. 1: Mineralien-Koffer für Schulen

zum Ziel, die Montanuniversität Leoben in der Erhaltung ihrer hohen Bildungsqualität zu unterstützen, diese durch internationale Kooperationen auszubauen und dabei die Attraktivität der Montanuniversität Leoben als internationale Bildungseinrichtung nach außen zu stärken.

Alle Bildungsaktivitäten des EIT RM streben danach, die Bewusstseinsbildung für Rohstoffe und deren nachhaltige Verwendung im modernen Alltag voranzubringen sowie eine ganzheitliche Sicht auf Rohstoffe zu vermitteln, welche sich an Kreislaufwirtschaft und Industrie orientiert. Dabei lassen die Rohstoffprojekte des EIT RM Bildung, Wissenschaft und Industrie im schulischen sowie akademischen Kontext zusammenfließen, wodurch den Lernenden verschiedene Karrieremöglichkeiten gezeigt werden. Sogenannte "T-shaped professionals" werden ausgebildet, die sich in bestimmten Bereichen hoch spezialisieren, sich aber auch gleichzeitig interdisziplinär zu bewegen wissen, wodurch ihre Beschäftigungsfähigkeit enorm gefördert wird. Lebenslanges Lernen wird dabei unumgänglich, um mit den raschen Veränderungen und Entwicklungen auf dem Arbeitsmarkt professionell mithalten zu können.

\section{Bildungsaktivitäten im schulischen Kontext zur Nachwuchsförderung und Stärkung des nachhaltigen Konsums von Rohstoffen in der Gesellschaft}

Im schulischen Kontext leisten die vom EIT RM geförderten Schulprojekte, wie zum Beispiel „RawMaterials@Schools" (http://rmschools.isof.cnr.it [3]) , "Briefcase” (https://youtu. be/GA8bHjefBfc [4]), "RAISE”, "RAISESEE“ sowie das teilgeförderte Projekt "BetterGeo" (https://www.sgu.se/ en/geology-of-sweden/bettergeo-minecraft-with-moregeology [5]) von der Primar- bis zur Sekundarstufe einen bedeutenden Beitrag zur Entwicklung eines hochwertigen und zeitgemäßen Unterrichts in den naturwissenschaftlichen Fächern.

Dabei soll einerseits die wissenschaftliche Begeisterung der Schülerinnen und Schüler für Rohstoffe geweckt und andererseits sollen Schüler/innen verstärkt im verantwortungsvollen Umgang mit Rohstoffen geschult werden. Neue Technologien werden dazu eingesetzt, um das Lernen kontinuierlich zu verbessern, um den Zugang zu Information und Bildung zu erleichtern, um die Zusammenarbeit von Schulen und Universitäten in der Forschung zu stärken und um Wissen zu teilen. Ein Einblick in die Praxis von Industrie und Wissenschaft soll Schüler/innen zukünftige Jobmöglichkeiten aufzeigen und die Auswahl von Studien aus dem Rohstoffbereich erleichtern.

Schulen sind sensible und dynamische Ökosysteme, die den stetigen Entwicklungen und Veränderungen des modernen Alltags und der Gesellschaft unterliegen. Obwohl davon auszugehen ist, dass alle europäischen Lehrpläne für die naturwissenschaftlichen Fächer die Auseinandersetzung mit Rohstoffen integrieren, findet die Adaptierung von Schulbüchern üblicherweise zeitverzögert von den technologischen Entwicklungen und Innovationen statt. Um daher mit Wissenschaft und Technologie mithalten zu können, müssen Lehrende oftmals zusätzliches Material für den Unterricht selbst entwerfen. Die Informationen dazu beziehen sie aus verschiedenen Quellen.

Zu diesen Quellen zählen das EIT RawMaterials und seine RawMaterials Academy. Schulische Projekte, wie zum Beispiel „RawMaterials@Schools“, „Briefcase”, „RawMaterials Students Internships“ (RAISE), „RawMaterials Students Internships in East South East Europe" (RAISESEE) und "BetterGeo" kommen hier zur Geltung. Diese Projekte richten sich an Schüler/innen und Lehrer/innen und laden zur intensiven interdisziplinären Auseinandersetzung mit den wissenschaftlichen, ökologischen, technischen, wirtschaftlichen, ethischen, sozialen und unternehmerischen Aspekten von Rohstoffen ein: Die Projekte bieten spannende Anregungen und hochwertige Materialien für den nachhaltigen Unterricht, wodurch die Lehrpersonen entlastet bzw. tatkräftig unterstützt werden sollen (Abb. 1).

In diesen Projekten werden im Unterricht erprobtes Material und ergänzende pädagogische Konzepte für den Umgang mit Rohstoffen entwickelt. Beispielsweise wurden/werden im Projekt „RawMaterials@Schools" und "Briefcase" Materialien erstellt, sogenannte "tool-kits" zu rohstoffrelevanten Themen, welche von Hintergrundinformationen, Versuchsanleitungen und „lesson plans" für die Lehrpersonen begleitet werden und dazu gedacht sind, im Unterricht eingesetzt zu werden. Zahlreiche Praxisbeispiele sollen dazu motivieren, die Schüler/innenbeteiligung an nationalen und internationalen wissenschaftlichen Veranstaltungen, wie zum Beispiel an der „Langen Nacht der Forschung" (www.langenachtderforschung.at) oder am jährlich in einem anderen europäischen Land stattfindenden „RawMaterials University Day“ des EIT RM (https:// eitrawmaterials.eu/events/rmud-2018-university-padova [6]) anzukurbeln. Um Lehrer/innen der naturwissenschaftlichen Fächer in der Umsetzung der Materialien optimal zu begleiten, finden internationale Workshops statt, in denen sich Lehrer/innen zusammen mit den Fachexpert/ innen austauschen können. Dabei fließen auch Expertise und Erfahrungen der teilnehmenden Lehrer/innen ein und die Materialien können gegebenenfalls adaptiert und optimiert werden. Die Ergebnisse werden in einer Datenbank gesammelt (Virtual Centre https://rmschools.isof.cnr. it/moodle [7]) und stehen der Bildungsgemeinschaft als Download zur Verfügung. 


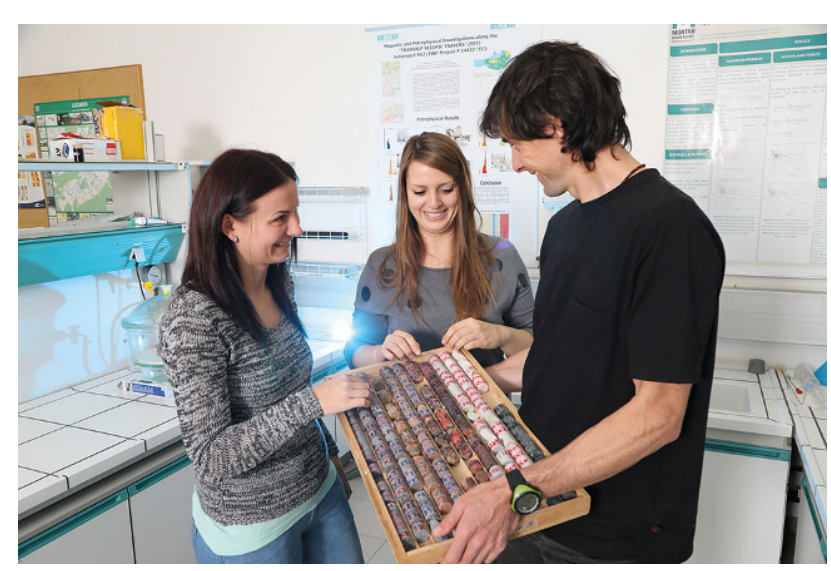

Abb. 2: Bohrkern-Studium an der Montanuniversität

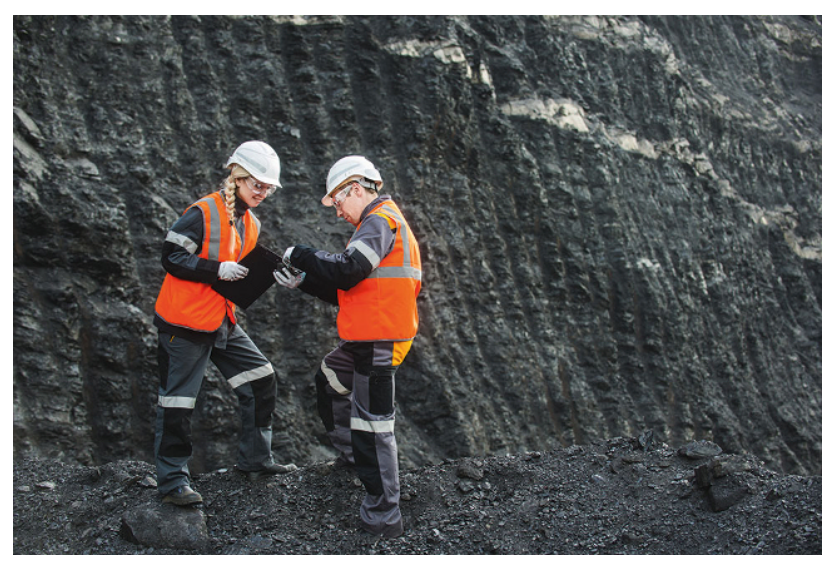

Abb. 3: Beurteilung im Tagebau

Neben der Materialentwicklung bzw. der wissenschaftlichen Auseinandersetzung mit naturwissenschaftlichen Inhalten wird in den Schulprojekten des EIT RM versucht, die Brücke zwischen Schule und Industrie zu schlagen. Zusätzlich zu Exkursionen und Schnuppertagen werden derzeit in zwei KAVA-Projekten (RAISE und RAISESEE) nationale und internationale Praktika organisiert, welche auf einem gemeinsamen pädagogischen und inhaltlichen Konzept aufbauen und sowohl den Schüler/innen als auch den Industriepartnern eine qualitätsvolle Begleitung bieten wollen.

\section{Bildungsaktivitäten im Hochschulbereich}

In diesen Aufgabenbereich fallen die Entwicklung und Implementierung neuer internationaler Hochschullehrgänge sowie internationaler Master- und PhD-Programme.

Aktuell arbeitet das RIC Leoben an der Einrichtung eines neuen internationalen MA-Programmes mit dem Titel "Innovation \& Entrepreneurship in Advanced Mineral Resources Development (ADMiReD)“, welches aus dem bereits bestehenden MA-Programm Advanced Mineral Resources Development (AMRD) an der Montanuniversitaet Leoben und der TU Bergakademie Freiberg entstanden ist. Beiden Programmen liegt die Auseinandersetzung mit nachhaltigen und umweltfreundlichen Methoden zur Roh- stoffgewinnung zu Grunde. Der Mehrwert des Programms ADMiReD besteht jedoch in seiner Schwerpunktsetzung auf den Themen "Innovation und Entrepreneurship" im Rohstoffsektor. Das heißt, zusätzlich zur Beschäftigung mit wirtschaftlichen, ökologischen und sozialen Aspekten des modernen Bergbaus widmet ADMiReD ein Semester innovativen und unternehmerischen Themen mit Bezug auf den Rohstoffsektor und leistet damit einen Beitrag zur Ausbildung von hochqualifizierten „T-shaped“ Rohstoffingenieur/ innen (Abb. 2).

\section{Berufsbegleitende Bildungsaktivitäten}

Zu den berufsbegleitenden Angeboten des EIT RM, die vom RIC Leoben betreut werden, zählen unter anderem die „CLC East Professional School“ (ECLCProSchool; https:// weiterbildung.tu-freiberg.de/TUF/search.xhtml) und die "Dubrovnik International Mining School" (DIM ESEE; https://dim-esee.eu). Diese Bildungsaktivitäten richten sich an Personen, die bereits im Berufsleben stehen, sich in ihrem Fachgebiet vertiefen wollen und/oder einen Branchenwechsel planen.

Beispielsweise bietet die ECLCProSchool, ein Projekt der Kategorie "Lebenslanges Lernen", ein Weiterbildungsprogramm zur Stärkung und Verbesserung der Professionalisierung von Fachpersonal in der Industrie. Die Kursinhalte basieren auf einer Bedarfserhebung in der ESEE-Region, die von der TU Bergakademie Freiberg mittels Umfrage durchgeführt wurde. Das Kursprogramm besteht aus einer Reihe ein- bis zweitägiger Workshops und Seminare zu verschiedenen Themen. Die ECLCProSchool möchte sich bis zum Projektende 2019 als eigenständige Bildungsmarke etablieren und kontinuierlich rohstoffrelevante Kurse für die Weiterbildung von Rohstoffingenieur/innen anbieten (Abb. 3).

Hauptziele der DIM ESEE, einem Projekt der Kategorie "RIS/INT", sind der Wissenstransfer sowie die Stärkung von Innovationskapazitäten im Bergbau, im Recycling und in der Abfallentsorgung.

Die DIM ESEE bietet ein einwöchiges internationales Weiterbildungsprogramm zur Verbesserung der Beschäftigungsfähigkeit von Ingenieur/innen in den verschiedenen Bereichen des primären und sekundären Rohstoffsektors. Langfristig möchte die DIM ESEE dazu beitragen, die Auswirkungen der Rohstoffgewinnung auf die Umwelt zu reduzieren, eine höhere gesellschaftliche Akzeptanz für den Bergbau zu erreichen sowie das wirtschaftliche Wachstum und die Beschäftigungsmöglichkeiten in den betroffenen Ländern zu verbessern.

Funding. Open access funding provided by Montanuniversität Leoben.

Open Access Dieser Artikel wird unter der Creative Commons Namensnennung 4.0 International Lizenz (http://creativecommons.org/licenses/ by/4.0/deed.de) veröffentlicht, welche die Nutzung, Vervielfältigung,

Bearbeitung, Verbreitung und Wiedergabe in jeglichem Medium und Format erlaubt, sofern Sie den/die ursprünglichen Autor(en) und die Quelle ordnungsgemäß nennen, einen Link zur Creative Commons Lizenz beifügen und angeben, ob Änderungen vorgenommen wurden. 
Hinweis des Verlags. Der Verlag bleibt in Hinblick auf geografische Zuordnungen und Gebietsbezeichnungen in veröffentlichten Karten und Institutsadressen neutral.

\section{Literatur}

1. European Innovation Partnership on Raw Materials: Raw Materials Scoreboard. https://publications.europa.eu/en/publication-detail/-/ publication/1ee65e21-9ac4-11e6-868c-01aa75ed71a1 (14.01.2019)
2. http://www.ehea.info (28.01.2019)

3. http://rmschools.isof.cnr.it (28.01.2019)

4. https://youtu.be/GA8bHjefBfc (28.01.2019)

5. https://www.sgu.se/en/geology-of-sweden/bettergeo-minecraftwith-more-geology (28.01.2019)

6. https://eitrawmaterials.eu/events/rmud-2018-university-padova (28.01.2019)

7. https://rmschools.isof.cnr.it/moodle (28.01.2019) 